Marcus Cyron

\title{
Wikipedia-Archäologie. \\ Zwischen Anspruch und Wirklichkeit
}

Vor einigen Jahren wurde zum ersten Mal eine Analyse des Zustandes der archäologischen Wissenschaften in der deutschsprachigen Wikipedia versucht. ${ }^{1}$ Während der Zeitraum zwischen 2009 und 2015 in der „normalen“ Welt als nicht lange erscheint, ist es im Internet und noch mehr in der Wikipedia ein immens langer Zeitraum, der eine erneute Betrachtung rechtfertigt. ${ }^{2}$

\section{Die archäologische Welt}

Auf die allgemeine Geschichte und Struktur der Wikipedia soll an dieser Stelle nicht eingegangen werden, das wurde schon an vielen anderen Stellen getan. Laut der internen Statistik weist die deutschsprachige Wikipedia zum 11. Februar 2015 genau 24.370 Einträge im Bereich der Archäologie aus. Erster Artikel war der Artikel „Archäologie“, der am 9. März 2002 von einem anonymen Benutzer angelegt wurde. Seitdem haben 326 angemeldete und 209 anonyme Benutzer fast 1000 Änderungen am Artikel vorgenommen. Er hat sich von einer ursprünglichen Größe von 663 Zeichen (Bytes) mit der Kernaussage „Archäologie ist die Lehre früherer Kulturtätigkeit des Menschen, durch Rekonstruktion der Umweltbedingungen und der Gesellschaftsordnung, hauptsächlich durch ihre physikalischen Überbleibsel (etwa Siedlungsreste, Keramik, Münzen usw.). Archäologie und Geschichte beeinflussen sich oft gegenseitig um einen möglichst breiten Blickwinkel auf die menschliche Geschichte zu ermöglichen" zu einem weitaus umfangreicheren Artikel mit 48.338 Bytes vergrößert. Doch jeder Fachmann, selbst der interessierte Laie mit ein wenig Grundwissen, der einen Blick auf den Artikel wirft, erkennt sofort einige Schwachstellen. Anders als viele andere Artikel ist er wirklich von der viel beschworenen „Schwarmintelligenz“ in vielen kleinen Stücken von verschiedenen Autoren verfasst worden. So fehlt ihm eine ordnende

1 Marcus Cyron: Eine Archäolopedia? Archäologie in der Wikipedia, In: Archäologisches Nachrichtenblatt 13 (2009), S. 293-299.

2 Vielen Dank an meinen Freund Jonathan Groß für seine Korrekturen und Anmerkungen zu diesem Beitrag. Ebenso Dank an Thomas Wozniak, der mir nicht nur die Gelegenheit gab, diesen Beitrag zu verfassen, sondern ihn auch kompetent in der Entstehung begleitete und mit seinen Hinweisen und Korrekturen sehr hilfreich war. Was an Fehlern verblieben sein sollte, ist allein mein Verschulden. 
Hand und er ist selbst in besseren Bereichen nur Stückwerk. Wie es um den zentralen Artikel steht, sind auch viele Bereiche und viele speziellere Artikel in der Wikipedia oft nur Fragmente. Aber es gibt natürlich auch Lichtblicke. Und glücklicherweise betreffen diese durchaus auch archäologische Teilbereiche.

Aber nähern wir uns dem Thema über die traditionellen akademisch verankerten Teildisziplinen:

Besonders kritisch steht es um die Ur- und Frühgeschichte. In kaum einem anderen Fachbereich ist die Abhängigkeit von einzelnen Autoren so eklatant sichtbar geworden wie in diesem. Als am 22. Januar 2011 der Mitarbeiter, der unter dem Pseudonym „Geos“ als Administrator und Autor seit 2004 in den Bereichen Geschichte des Römisches Reiches sowie Provinzialrömische und Prähistorische Archäologie tätig, nach längerer Krankheit verstarb, war das Loch, das er hinterließ, immens groß. Zudem war er die Person, die dem ganzen Bereich als freiwilliger Betreuer Struktur verliehen hat. Das konnte durch korrekte Kategorisierungen der Artikel erfolgen, durch die Betreuung neuer Autoren oder in anderen Formen. Problematisch wurde es zudem, als ein Hobbyforscher seine eigenen Erkenntnisse zu Megalithkulturen in der Wikipedia zu bewerben begann. Seine Artikel waren einerseits äußerst umstritten, andererseits konnte keiner der anderen in der Archäologie bewanderten Autoren diese wirklich beurteilen. Niemand hatte das entsprechende Fachwissen und lange blieb ein flaues Gefühl. Was blieb, war das Einholen externer Gutachten bei universitären Archäologen, ${ }^{3}$ die kaum ein gutes Haar an diesen Artikeln ließen. All das führte zu einer Sperre des betreffenden Autoren, die gut dreieinhalb Jahre Bestand hatte. Seit der Entsperrung und „Wiedereingliederung“ hat sich die Qualität der Beiträge merklich gebessert, der wikipedianisch-enzyklopädisch-neutrale Standpunkt hat mittlerweile Einzug gehalten. Umstritten blieben die Artikel dennoch weiterhin sowohl innerhalb der Wikipedia als auch nach Berichten von externen Fachleuten außerhalb der Wikipedia.

Mittlerweile ist die Ur- und Frühgeschichte Mittel-, Süd- und Westeuropas schon in weiten Teilen mit wichtigen Artikeln vertreten, so fehlen kaum noch Hauptartikel, wenngleich die Qualität vielfach zu wünschen übrig lässt. Doch schon in Ost- und Südosteuropa (abgesehen vom südlichen Balkan) werden die Lücken größer. Für Asien und die Amerikas gibt es immer wieder besser erschlossene Bereiche, doch meist bestimmen die Lücken das Bild. Sind in Europa vor allem archäologische Kulturen recht gut erschlossen und fehlen oft archäologische Fundplätze, ist es bei Artikeln zu Themenbereichen außerhalb Europas

3 In diesem Fall von Ur- und Frühgeschichtlern des mittlerweile abgewickelten Ur- und Frühgeschichtlichen Instituts der Humboldt-Universität zu Berlin. 
genau anders herum. Problematisch sind oft die Artikel von „Quereinsteigern“. Damit sind nicht die Autoren gemeint, die ihr Wissen im Selbststudium und nicht an einer Hochschule erworben haben, die im Gegenteil oft eine tiefe Durchdringung von Themen in der Wikipedia erreichen, also „Dilettanti“ im besten Sinne des Wortes sind, sondern die Autoren, die sich beispielsweise mit der Geschichte der Waffentechnik beschäftigen, und aus einem gewissen Drang zur Komplettierung in Bereichen arbeiten, von denen sie wenig bis keine Ahnung haben, für das sie oftmals nicht einmal ausreichendes Verständnis mitbringen. In solchen Fällen kommt es regelmäßig zu Konflikten.

Ein vergleichsweise gut aufgestellter Bereich ist die Ägyptologie. Es ist eines der wenigen Themengebiete, in dem es derzeit eine aktive, miteinander arbeitende Wikipedia-Community gibt, selbst wenn es auch hier nicht immer reibungslos läuft. Ägyptologie wird im weitesten Sinne der Disziplin verstanden, wenngleich die klassischen Kernthemen auch für die Wikipedia-Autoren den Zeitrahmen von der prädynastischen Zeit bis in die Spätzeit umfassen. Ähnlich wie die wissenschaftliche Forschung nimmt die Beschäftigung mit der vorschriftlichen Zeit immer mehr zu. Es gelingt den Wikipedia-Autoren anders als etwa der universitären Landschaft in Deutschland eine recht ausgewogene Sicht auf Altägypten zu vermitteln. Entgegen dem Trend an deutschen Universitäten, die die Archäologie Altägyptens nahezu nicht mehr in der Lehre anbieten, sondern nur noch ein rein philologisches Fach repräsentieren, stehen in der Wikipedia gleichrangig archäologische und kunsthistorische Artikel neben denen zu Sprache, Literatur und Geschichte. Im Zentrum der Autoren stehen neben biografischen Artikeln zu Herrschern und Beamten die „klassischen“ Artikel zu den Pyramiden, den Tempeln sowie den Grabanlagen. Diskussionen zu Chronologie und (altägyptischen) Schreibweisen sind an der Tagesordnung.

Weit weniger gut steht es um die Vorderasiatische Archäologie. Hier war der Bereich, wie auch die Ur- und Frühgeschichte, von einem Autor abhängig. Dieser Mitarbeiter, der unter dem Namen „NebMaatRe“ auftrat, verstarb Anfang Juli 2011. Und auch hier fiel, wie in der Ur- und Frühgeschichte, damit die ordnende Hand weg. Zwar fand sich wenig später ein ähnlich kompetenter Mitarbeiter für den Bereich der mesopotamischen Kulturen, dem allerdings sein eigenes Ungestüm im Wege stand und er deshalb Probleme hatte, mit anderen Mitautoren zusammen zu arbeiten. Somit ist dieser Bereich zumindest für die Gebiete des heutigen Iraks, Syriens, Libanons und Jordaniens derzeit unbetreut. Natürlich entstehen auch hier immer wieder Artikel und es werden auch immer wieder Artikel bearbeitet, erweitert, ergänzt und korrigiert. Es scheinen jedoch zumeist Artikel zu sein, die weniger zielgerichtet entstehen. Besser ist die Situation für Altvorderasien. Auch wenn es niemanden gibt, der hier eine Führungsrolle übernimmt, hat Wikipedia mit einem Autoren unter dem Pseudonym „Kpisimon“ einen Mitarbeiter, der sich 
um alle neuen Artikel kümmert. Andere Bereiche, wie etwa der Altsüdarabiens, stehen etwas besser da als der Schnitt, weil sich mit dem Benutzer „Schreiber“ ein Autor gefunden hat, der diesen ausführlicher bearbeitet. Der Altiran steht besser mit seinen historischen Artikeln da als mit seinen archäologischen.

Der wohl umfangreichste archäologische Themenbereich in der deutschsprachigen Wikipedia ist der der „Klassischen“ Antike. Obwohl Ur- und Frühgeschichte sowie Mittelalterliche und Neuzeitliche Archäologie die deutschsprachigen Leser in der Praxis eher betreffen und obwohl die Ägyptologen seit längerer Zeit besser organisiert sind, dürfte in der Breite das griechisch-römische Altertum in "guter deutscher“ Tradition besser dastehen als alle anderen Bereiche zusammen. Seit ich in der Wikipedia tätig wurde, gab es keinen archäologischen Bereich, der auf Dauer von so vielen Autoren profitieren konnte. Deshalb dürfte keiner der Hauptartikel mehr fehlen, wenngleich auch hier die Qualität sehr divergiert. Je mehr Interesse ein Autor am Thema hatte, je mehr er davon verstand, desto besser ist im Schnitt auch der Artikel. Das mag wie ein Allgemeinplatz klingen, ist aber für das generelle Verständnis der Wikipedia und ihrer Autoren von eminenter Bedeutung und kann gar nicht oft genug betont werden. Und das setzt sich auch nach unten, in die Breite der Themen fort. So dürfte in aller Bescheidenheit kaum ein Bereich in einer solchen Breite bearbeitet worden sein, wie der zur antiken griechischen Keramik, in dem ich als der Hauptautor gelten dürfte. Darüber hinaus zeigt sich ein Bild der verschiedenen Autoreninteressen auf der einen, von der Zugänglichkeit zu Informationen (die sicher verschiedene Gründe hat, aber auch sehr mit deutscher Tradition zu tun hat) auf der anderen Seite zu tun hat. So entstehen eher Artikel über antike Bauwerke in Rom, Athen oder Pompeji als zu weniger bekannten Orten. Klassische deutsche oder österreichische Grabungen sind eher auch über Wikipedia-Artikel erschlossen als Grabungen anderer Länder, vor allem dann, wenn die Publikation eher über weniger verbreitete Sprachen erfolgt. Dass zuletzt etwa ein Aufschwung in der Behandlung nordafrikanischer Themen erfolgte, kann man neben persönlichen Interessen wohl auch dem verstärkten Engagement des DAI in Nordafrika zuschreiben, das eben auch eine verstärkte Publikationstätigkeit in diesem Bereich im deutschsprachigen Raum mit sich bringt.

Nicht nur aus der Erkenntnis, dass das Verständnis solcher Kulturen auch immer ein Verständnis ihrer Erforscher und deren Zeitumstände beinhaltet, ist ein weiterer wichtiger Punkt die Beschäftigung mit der Fachgeschichte. Schon 2008 begann ich mit meinem Freund Jonathan Groß ein Projekt, über das wir die Wissenschaftler in den Altertumswissenschaften auch über die Archäologien hinaus zusammenstellten, Lehrstühle in ihrer Entwicklung verfolgten und so weiter. Im Endergebnis haben mittlerweile alle aktuellen wie auch ehemaligen Lehrstuhlinhaber im Fachbereich der Klassischen Archäologie im deutschspra- 
chigen Raum Wikipedia-Artikel. In der Alten Geschichte fehlen aktuell noch Artikel zu zwei Personen, in der Klassischen Philologie sind die Lücken aufgrund der längeren Tradition der Lehrstühle noch etwas größer, wenngleich auch hier seit dem 19. Jahrhundert eine weitgehende Artikeldeckung vorhanden ist. Dank mehrerer Mitarbeiter um Jonathan Groß, der derzeit Gymnasiallehrer des 19. und frühen 20. Jahrhunderts bearbeitet, ist auch die Personengeschichte des PaulyWissowa recht gut erschlossen. Durch Autoren wie „Tusculum“ oder „Korrekturen“ konnte der Blick über den deutschsprachigen Tellerrand hinaus zuletzt stark geschärft werden. Franzosen, Schweden, Finnen, Italiener - aus vielen Nationen fanden die Biografien von Archäologen mittlerweile Einzug in das Lexikon. Derzeit ist ein Artikel, der all das zusammenführt und die Geschichte der Altertumswissenschaft(en) nachzeichnet, allerdings noch ein Desiderat.

Lange Zeit äußerst aktiv und selbst von Fachleuten außerhalb der Wikipedia sehr geschätzt, war der Bereich der Provinzialrömischen Archäologie. Die Mitarbeiter um die Hauptautoren Hartmann Linge (Westdeutschland), Mediatus (Süddeutschland) und Veleius (Österreich) hatten ganze Limesabschnitte bearbeitet. Kontakte bestanden weit über die Grenzen des deutschen Sprachraums hinaus. Niederlande, Ungarn, Nordafrika - überall konnten die Mitarbeiter des Limes-Projektes Hilfe finden. Zwischen 2008 und 2012 gab es organisierte Treffen, 2008 auf der Saalburg, 2009 schon über zwei Tage in Köln und Xanten, 2009 in Österreich und 2012 schließlich über mehrere Tage in Hessen. Doch auch hier konnte man einmal mehr sehen, dass Erfolg und Misserfolg nicht selten von einer Person, abhängen. Dieses mal war es keine Person die verschwand, sondern eine, die dazu kam und mit ihrer Art das bis dahin sehr freundschaftlich und kollegial verlaufende Projekt sprengte.

Andere archäologische Bereiche stehen eher schlecht da. Das europäische Mittelalter und die Neuzeit werden abgesehen von vereinzelten Artikeln kaum bearbeitet. Hier gibt es allerdings auch sehr enge Überschneidungen mit der Kunstgeschichte. Wenn sich ein Autor aus Interesse am deutschen Steinzeug mit diesem etwas eingehender befasst, erbringt das neben der Bearbeitung eines kunsthistorisch-kunsthandwerklichen Themas manchmal eben auch am Rande die Beschäftigung mit neuzeitlicher Archäologie mit sich. Zielgerichtet verläuft diese Arbeit jedoch nicht. Einzelaktionen führten zu einer punktuellen Bearbeitung von Themengebieten in Südostasien und Subsahara-Afrikas. Wenn noch ein Gebiet heraus gehoben werden kann, ist es die Altamerikanistik. Nicht eigentlich eine Archäologie, sondern an den deutschen Universitäten ein ethnologisches Fach, bedient sie sich aber dennoch vieler archäologischer Techniken. Auch hier entstanden zu den Hauptkulturen und ihren Hauptorten in Mittel- und Südamerika schon viele Artikel. Einer der Hauptautoren war dabei bis zu seinem Ableben im Jahr 2014, wie in der Autorengemeinschaft erst etwas später realisiert wurde, 
der ehemalige Professor Hanns J. Prem. Seine Mitarbeit war konfliktfrei und ganz einfach nur von guter Artikelarbeit geprägt. Er gehörte damit zu den vielen leisen Autoren, die in der Wikipedia aus Freude am Schreiben und aus Freude an der Weitergabe des Wissens mitarbeiten, ohne seine Person besonders wichtig zu nehmen. Solche Autoren hat Wikipedia leider noch zu wenig, glücklicherweise gibt es sie aber - und mittlerweile auch in der Archäologie - immer öfter.

Mittlerweile verändern sich die Formen der Wikipedia-Arbeit. Hatte man früher seinen Computer, dazu im Idealfall ein oder mehr Bücher als Grundlage und einen Artikel als Ziel, gibt es nun vielfach ganz anderen Anstöße. So hat sich in den letzten Jahren international der sogenannte „GLAM“-Bereich heraus gebildet. In diesem - GLAM ist ein Akronym und steht für „Galleries, Libraries, Archives and Museums“ - wird oft sehr zielgerichtet die Zusammenarbeit mit Institutionen aus diesen Bereichen gesucht. In Deutschland findet diese Zusammenarbeit bislang vor allem mit Museen statt. Zunächst gab es im September 2012 Versuche mit dem Museumsdorf Düppel in Berlin, leider kam man über erste Versuche der Annäherung nicht hinaus. 2013 schließlich wurden Wikipedianer an das Braunschweiger Landesmuseum eingeladen, um dort die Ausstellung zum Harzhorn-Ereignis zu begleiten. ${ }^{4}$ Andere Kontakte gibt es etwa zum Museum August Kestner in Hannover, wo Wikipedianer eine Führung im Rahmen eines ihrer GLAM-Treffen bekamen, oder mit der Archäologischen Zone in Köln, wo es ebenfalls im Anschluss an Probleme mit dem entsprechenden Artikel in der Wikipedia eine Führung für Wikipedianer gab. Die Probleme rührten daher, dass von Mitarbeitern der Archäologischen Zone der Artikel in einer Weise bearbeitet wurde, wie es den Wikipedia-Regeln entgegen steht. Da es hier aber weder von der einen, noch der anderen Seite eine böse Absicht gab, konnte man sich gütlich zusammentun und den Artikel mit Hilfe der Kölner Archäologen auf einen angemessenen Stand bringen, was aufgrund der generell problematischen Sachlage in diesem Forschungsbereich nicht leicht war und interessanterweise nicht zuletzt durch die Hilfe fachfremder Autoren, die einen neutraleren Blick hatten, zustande kam.

\footnotetext{
4 In Vorbereitung auf die Landesausstellung „Die Römer kommen“, die sich den neuen Funden eines militärischen Aufeinandertreffens am Harzhorn widmete, wurden Wikipedia-Autoren für ein Wochenende ins Museum eingeladen um einerseits Artikel zum Thema zu verfassen, andererseits konnten sie aber auch Vorbereitungen der Ausstellung begleiten, bekamen Führungen hinter die Kulissen des Museums und wurden von den verantwortlichen Archäologen am Ort der Schlacht geführt. Aufgrund des Erfolges der Veranstaltung wurde sie mit der Ausstellung zum Ersten Weltkrieg in Braunschweig wiederholt.
} 


\section{Wikipedian in Residence beim DAI}

Auch wissenschaftliche Institutionen wie das Deutsche Archäologische Institut (DAI), seines Zeichens die größte archäologische Institution der Welt, arbeiten mit Wikipedia, insbesondere mit Wikimedia Deutschland, dem Unterstützerverein für die Wikimedia-Projekte in Deutschland, zusammen. Im Rahmen dieser Zusammenarbeit kam es auch zur ersten Beschäftigung eines „Wikipedian in Residence“ beim DAI. Hierbei repräsentiert der Resident zum einen die Wikimedia-Projekte in der Institution, zum anderen diese aber auch gegenüber den Wikimedia-Projekten. Die genaue Ausgestaltung kann sehr unterschiedlich sein, der Begriff „Wikipedian in Residence“ ist auch keine geschützte Berufsbezeichnung. Hier kann man sich nach den Bedürfnissen aller Beteiligter richten. Ich selbst hatte das Glück, diese Position als erster offizieller „Resident“ in Deutschland in der zweiten Hälfte des Jahres 2012 beim DAI bekleiden zu dürfen. Hierbei versuchte ich zunächst den Mitarbeitern des DAI Wikipedia näher zu bringen. Das erfolgte zum einen über einführende Vorträge, zum anderen auch über ganz praktische einführende Workshops in die aktive Wikipedia-Arbeit. Dabei hatte ich das Glück, mich nicht nur auf die Berliner Zentrale beschränken zu müssen, sondern konnte auch vier der Außenstellen des DAI, in Athen, Rom, Madrid und Istanbul besuchen. Ob ich einen der Archäologen, Bauforscher oder anderen Mitarbeiter zu einem „Wikipedianer“ machen konnte, weiß ich nicht, aber ich denke, dass es nicht umsonst ist, wenn Wissenschaftler auch nur wissen, wie diese Wikipedia, die häufig auch für sie recht wichtig geworden ist, funktioniert. Das Interesse an einer Mitarbeit war zum Teil deutlich spürbar, von jungen Archäologen habe ich auch gehört, dass sie auch gerne etwas in der Wikipedia in der Sprache ihres Gastlandes getan hätten. Leider musste ich auch sehr häufig hören, dass Zeit ein hinderlicher Faktor ist. Institutionen, die sich verstärkt bei Wikipedia engagieren wollen und nicht nur einen „Wikipedian in Residence“ als Autoren suchen, Institutionen, die möchten, dass ihre Mitarbeiter wissen, wie Wikipedia funktioniert, sollten diesen auch eine Mitarbeit in der Arbeitszeit zugestehen. Möglicherweise hat nur das einen längeren Effekt. Vielleicht gibt es aber auch noch Ideen, die bisher niemandem kamen. Hier sollten mögliche Interessenten aus den verschiedenen Institutionen mit ihren Ideen einfach anfragen, für fast alle Ideen findet sich sicher auch der eine oder andere Wikipedia-Autor. Zentraler Ansprechpartner ist derzeit der Unterstützerverein der Wikimedia-Projekte in Deutschland, der „Wikimedia Deutschland e.V.“. Wer keinen Autoren gefunden hat, den er gerne ansprechen wollte, kann den Verein als Wegweiser nehmen. Wikimedia Deutschland unterstützt gegebenenfalls auch aktiv eine Zusammenarbeit, auch hier kommt es immer darauf an, was ein „Wikipedian in Residence“ tun soll. Nur eines ist klar: tradi- 
tionell kann Wikimedia Deutschland schwerlich Projekte aktiv unterstützen, bei denen ein „Resident“ in der Arbeitszeit Artikel schreiben soll.

\section{Wikipedia trifft Institutionen}

Ein anderer Ansatz aus der Wikipedia hinaus mit wissenschaftlichen Institutionen in Kontakt zu treten war die Tagung „Wikipedia trifft Altertum“, die 2011 von Martin Rulsch, Jonathan Groß und mir mit Unterstützung aller wichtigen Fachund Berufsverbände im Bereich der Altertumswissenschaften an der Universität Göttingen organisiert wurde. Hier kamen 100 Vertreter verschiedener Altertumswissenschaften und Wikipedianer zusammen. Über die Hälfte der Teilnehmer kamen dabei aus wissenschaftlichen Institutionen. 27 Vorträge wurden von Professoren bis Nachwuchswissenschaftlern sowie von Wikipedianern über deren Wikipedia-Fachbereiche gehalten. ${ }^{5}$ Bis heute gilt die Tagung aus Sicht der Wikipedia als Meilenstein bei der Zusammenarbeit mit der „Scientific Comunity“, die sehr zum Verständnis beider Seiten füreinander beigetragen hat. Ihr folgte 2013 „Wikidata trifft Archäolgie“. Die Tagung ging als Abschluss und Höhepunkt aus meiner Zeit als „Resident“ beim DAI hervor und fand mit mehr als 50 Fachvertretern und Wikipedianern im Auswärtigen Amt in Berlin statt. Mittelpunkt war eine von Wikipedia-Autoren erstellte Karte, in der in interaktiver Weise die römische Reichsgrenze mit ihren Kastellen gezeigt wurde. Hierzu sollten die Daten auf dem zu der Zeit ganz neuen Datenlager Wikidata ${ }^{6}$ gesammelt werden. Leider mussten wir feststellen, dass ein weiterer wünschenswerter Ausbau der Karte auf rein ehrenamtlicher Grundlage nicht möglich ist. Eine weitere Arbeit an diesem an sich sehr lobenswerten Projekt kann nur mit entsprechender finanzieller Absicherung erfolgen.

Andere Institutionen haben in den letzten Jahren für das freie Medienarchiv Wikimedia Commons Dateien, insbesondere Bilder, gespendet. Das begann 2008 mit mehr als 80.000 Bildern des deutschen Bundesarchives, hier waren aber noch

5 Tagungsflyer: https://de.wikipedia.org/w/index.php?title=Datei:Flyer_Wikipedia_trifft_Alter tum.pdf\&page=2; Beschreibungsseite in der Wikipedia: https://de.wikipedia.org/wiki/Wikipe dia:Wikipedia_trifft_Altertum (15.3.2015).

6 Wikidata ist eine Ende Oktober 2012 gestartete Datenbank, in der zentral verschiedene Daten, beziehungsweise Aussagen gesammelt werden sollen. Sie soll zunächst einmal die WikimediaProjekte unterstützen, aber auch darüber hinaus nutzbar sein. Sie ist in der Form aufgebaut, dass jedem Eintrag bestimmte Funktionen zugewiesen werden können. Beispielsweise werden einem Eintrag „Theodor Mommsen“ Aussagen wie „Mann“, „Deutscher“, „Althistoriker“ oder „Träger des Nobelpreises für Literatur“ zugewiesen und können auch ausgewertet werden. 
kaum archäologische Bilder dabei. Auch die im März des folgenden Jahres erfolgten Großspenden des Amsterdamer Tropenmuseum (knapp 50.000 Bilder) und der Deutschen Fotothek (62.000 Bilder) brachten hier noch keine nennenswerten Zugänge im Bereich der Archäologien. Erst im Oktober 2010 konnte durch eine etwa 3.000 Bilder umfassende Spende des Muséum de Toulouse ein erster nennenswerter Zugang an Bildern in dem Bereich erreicht werden. Bislang den größten Zugang hatte Wikimedia durch die Spende von mehr als 18.000 Bildern des Walters Art Museums, darunter auch sehr viele archäologische Bilder. Vor allem in Deutschland sträuben sich noch viele Museen, überhaupt das Fotografieren zu erlauben, geschweige denn, dass bislang eines auf die Idee gekommen wäre, seine Bilder unter Freien Lizenzen zu veröffentlichen. Zum einen werden Einkunftsverluste befürchtet, zum anderen ist die Angst vor einem Verlust der Deutungshoheit noch vielfach vorhanden. Die Vorbehalte sind einerseits verständlich, andererseits gab es weder je diese Deutungshoheit noch kann man vielfach heute noch Steuerzahlern, die den Großteil der Museen und Sammlungen mit ihren Steuern unterstützen oder fast ganz tragen, klar machen, warum sie nicht ein Anrecht darauf haben wenigstens ihre eigenen Bilder zu machen und mit diesen dann auch nach ihrem Willen zu verfahren. Hinzu kommt, dass neben der Finanzierung durch Steuern oft noch eine zweite Finanzierung durch den Eintritt erfolgt. Viele vor allem kleinere Museen, die ohnehin kaum Einnahmen vom Bilderverkauf haben, sträuben sich schon lange nicht mehr gegen solche Dinge, die weder in Zeiten von Smartphones Sinn machen, noch das mögliche Interesse abwürgen, das sich auch in dem Aufnehmen von Fotografien ausdrücken kann. Leider werden Bilder auf Wikimedia Commons vielfach mit nicht aussagekräftigen Beschreibungen hochgeladen und dann auch nicht in entsprechende Kategorien eingebunden. Somit befinden sich nicht selten ungenutzte, unnutzbare Bilder auf Commons. Die Bilder jedoch, die gut beschrieben und kategorisiert sind, sind nicht selten weit verbreitet, auch über Wikipedia und andere Wikimedia-Projekte hinaus.

\section{Fazit}

Was bleibt? Einerseits gibt es zumindest für den Raum Europas, des Mittelmeergebietes und Vorderasiens eine recht große Dichte an Artikeln, die zumindest für den Einstieg schon vieles abdecken. Inwieweit es dann in die Tiefe, in die Breite und in die Spitze geht, liegt an den entsprechenden Interessen der Autoren und ob sich überhaupt Autoren gefunden haben. Somit geht es der Archäologie in der Wikipedia ähnlich wie den meisten anderen Bereichen. Leider hatten Versuche, die Archäologie in der Wikipedia etwas strukturierter anzugehen und eine Art 
Redaktion zu schaffen, wie es etwa bei den Biologen oder Medizinern schon länger gut funktioniert, keinen nachhaltigen Erfolg. Zusammenarbeit erfolgt weiter eher zwischen einzelnen Autoren, als zielgerichtet innerhalb einer größeren Autorengruppe. Vor allem in der Qualitätssicherung ist das bislang unbefriedigend. Das Zukunftsmodell könnte womöglich in der Einbeziehung von außerwikipedianischen Institutionen liegen. Ganz persönlich würde es mich ja freuen, wenn in absehbarer Zeit eine archäologische Sammlung, gerne auch eine universitäre Sammlung, mit uns zusammenarbeiten würde. Wir könnten etwa bei der Erschließung von Beständen helfen, beim Anfertigen guter Fotos und Ähnlichem und würden dann die Ergebnisse gerne unter Freien Lizenzen veröffentlichen. 